\title{
Out of One, Many: Distinguishing Time Delays from Lensed Supernovae
}

\author{
Mikhail Denissenya, ${ }^{1 \star}$ Satadru Bag $^{2}$, Alex G. Kim ${ }^{3}$, Eric V. Linder ${ }^{1,3,4}$, Arman Shafieloo ${ }^{2,5}$ \\ ${ }^{1}$ Energetic Cosmos Laboratory, Nazarbayev University, Nur-Sultan 010000, Kazakhstan \\ ${ }^{2}$ Korea Astronomy and Space Science Institute, Daejeon 34055, Korea \\ ${ }^{3}$ Lawrence Berkeley National Laboratory, Berkeley, CA 94720, USA \\ ${ }^{4}$ Berkeley Center for Cosmological Physics, University of California, Berkeley, CA 94720, USA \\ ${ }^{5}$ Department of Astronomy and Space Science, University of Science and Technology, Daejeon 34113, Korea
}

25 February 2022

\begin{abstract}
Gravitationally lensed Type Ia supernovae are an emerging probe with great potential for constraining dark energy, spatial curvature, and the Hubble constant. The multiple images and their time delayed and magnified fluxes may be unresolved, however, blended into a single lightcurve. We demonstrate methods without a fixed source template matching for extracting the individual images, determining whether there are one (no lensing) or two or four (lensed) images, and measuring the time delays between them that are valuable cosmological probes. We find $100 \%$ success for determining the number of images for time delays greater than $\sim 10$ days.
\end{abstract}

Key words: gravitational lensing: strong - transients: supernovae-cosmology: observations - methods: numerical, data analysis

\section{INTRODUCTION}

Time delays in strong gravitational lens systems are one of the few dimensionful quantities that can be measured cosmologically. This enables an absolute distance measurement, rather than a ratio of distances, and so determines an absolute cosmic length scale, e.g. the Hubble constant (Refsdal 1964). The time delay distance formed from the time delay involves combinations of distances from observer to source, observer to lens, and lens to source; this is a valuable probe of both the cosmic expansion behavior and hence dark energy, and spatial curvature (Linder 2004, 2011; Wong et al. 2020; Millon et al. 2020; Shajib et al. 2020; Liao et al. 2020; Treu \& Marshall 2016).

Each strongly lensed source is split so as to appear as multiple images, with generally the central lensed image unobservable due to lens galaxy obscuration, leaving two or four images. These images will be magnified in flux, and delayed in time, relative to the source flux and each other. Type Ia supernovae (hereafter just SN) have particular advantages as gravitationally lensed sources, as they are time variable (so time delays are detectable), their intrinsic time variation is fairly well known, the observations needed to measure time delays span a month or two rather than years as for lensed quasars, and the SN distances are standardizable, simplifying the lens system modeling.

If the multiple images can be resolved, i.e. their angular separations are greater than the observing resolution, then each image can be measured separately. Some particular recent work on such lensed supernova cosmology appears in, e.g., (Huber et al. 2022; Rodney et al. 2021; Pierel et al. 2021). However, for worse image resolution, smaller lensing masses, or less favorable geometry of the lens and source positions, the images may overlap and blend to-

\footnotetext{
^ Email: mikhail.denissenya@nu.edu.kz
}

gether - be unresolved. In this case only a single combined lightcurve (flux vs time) can be measured, defining a system. We only use this observed lightcurve data.

We focus here on such unresolved lensed SN, investigating whether we can determine from a combined lightcurve its constituent elements: is it lensed or unlensed, how many images, and what are their time delays (and magnifications, to a lesser extent). For unresolved lensed supernovae this work covers the key range of 6-14 days motivated by the expected distribution of time delays generally peaked around 10 days as in, e.g., (Goldstein et al. 2019). This extends Paper 1 (Bag et al. 2021) in this series by going beyond two image systems to allow four images and a more robust exploration of multiplicity, and using a complementary fitting method, also without a fixed source template.

In Section 2 we describe the new fitting method and crosscheck it against both simulations and the previous method for two images. We analyze it for four image systems in Section 3 and quantify its precision, accuracy, and range of robustness against simulations. Section 4 investigates determination of multiplicity, i.e. distinguishing unlensed (one image) systems from two image from four image systems, presenting the "confusion matrix" between them. We summarize the current state and future work in fitting unresolved lensed $\mathrm{SN}$ in Section 5.

\section{FREEFORM FITTING}

Strong lensing creates multiple images, each delayed in time and magnified (greater or less) in flux. If the images can not be resolved, then the signal is blended, a superposition of all images. The resulting blended lightcurve defines a system. For an intrinsic, unlensed source 
flux $U(t)$, the measured, blended lightcurve is

$F_{j}(t)=\sum_{i \in \text { images }} \mu_{i} U_{j}\left(t-\Delta t_{i}\right)$.

Here $j$ indexes different wavelength filter bands, $i$ the different images, and $\mu_{i}$ and $\Delta t_{i}$ give the magnification and time delay, respectively, of image $i$. We aim to fit for $\Delta t_{i}$ and $\mu_{i}$ (though we are mostly interested in $\Delta t_{i}$ ); note these quantities are one (important) step among many to use strong lensing systems for cosmology. The freeform technique works strictly from the observed lightcurve data: supernova properties such as stretch, color, redshift do not enter further as the method is freeform, i.e. model agnostic. As in Paper 1, we do not include microlensing, which would turn the numbers $\mu_{i}$ into time and wavelength dependent functions $\mu_{i, j}(t)$. We address microlensing in future work, but here focus on a direct comparison to and extension of Paper 1.

If one knew the source time evolution $U(t)$ perfectly, then fitting for $\Delta t_{i}, \mu_{i}$ is straightforward. However, $\mathrm{SN}$ do vary in their lightcurve intrinsically, and any template adopted will lead to errors, and possibly biases, in the extracted time delays. Furthermore, we wish to keep our method reasonably general so it might be applicable in the future to other lensed transients besides Type Ia supernovae. Therefore we did not adopt a fixed template in Paper 1, and do not here.

\subsection{Free Within Bounds}

In Paper 1, we took a base lightcurve form with an asymmetric rise and fall (specifically a lognormal) and multiplied it with a Crossing hyper-function (fourth order polynomial constructed from the first four Chebyshev polynomials) (Shafieloo et al. 2011; Shafieloo 2012a,b; Hazra \& Shafieloo 2014). This gives considerable freedom for the lightcurve shape. However, it does correlate the shape at one time with that at another time (recall the Chebyshev polynomials represent a linear tilt, parabolic curvature, etc. as they increase in order). Therefore we also explored an alternate form allowing freedom in the lightcurve shape, which we use in this paper.

The technique here is of freedom at each time (SN phase), uncorrelated with other times. Complete freedom in the lightcurve shape would lead to a fully degenerate problem, so we impose bounds on the amplitude of the freedom. Concretely, we have

$U_{j}(t)=H_{j}(t)\left[1+h_{j}\left(t_{k}\right)\right]_{G S(t)}$,

where $H_{j}(t)$ is the Hsiao (Hsiao et al. 2007) template for Type Ia supernovae in wavelength band $j$. We use Zwicky Transient Facility (ZTF; Bellm et al. (2018)) $g, r, i$ bands. The fit parameters $h_{j}$ are hyperparameters embodying the freedom to change the lightcurve shape, but we bound their amplitude by $\left|h_{j}\right|<b$. We explored various values for the limiting fractional change $b$, and found that $b=0.1$, i.e. a $10 \%$ change was ample to cover the variation of SN intrinsic lightcurves used for cosmology, giving the best combination of freedom and accuracy.

The hyperparameters $h_{j}$ are independently chosen at nodes $t_{k}$ throughout the SN phase. The lightcurve in between nodes, i.e. the full $U_{j}(t)$, is formed by multiplying a Gaussian kernel smoothing in time of the $1+h_{j}\left(t_{k}\right)$ factor (denoted by the subscript $G S(t)$; the smoothing length is itself a hyperparameter, lying in $[3,8]$ days) by a linear spline of $H_{j}$ (the Hsiao template is defined at discrete epochs). We found this works better and is more computationally efficient than Gaussian smoothing (or splining) both factors. The number of nodes used is typically 50-70, depending on the observation range. Too small a number of nodes will not give sufficient freedom for variations, while too large a number of nodes will oversample the data as well as increase the computational cost. We experimented with both adaptive and equal time spacing, and found equal spacing to work well, avoiding the potential for adaptive spacing to become too sparse in certain epochs and miss useful features (e.g. troughs as well as peaks inform the time delay estimation).

As in Paper 1, we simulate data using our LCsimulator code based on the sNCOSMO (Barbary et al. 2020) PYTHON package, with lightcurves generated by applying noise and observing characteristics as in (Goldstein et al. 2019) to Hsiao spectra (see Appendix A for tests with alternative lightcurves). The modeled lightcurves in $g, r$, $i$ ZTF bands include observations with $\sim 2-4$ day cadences with the flux uncertainties set to $5 \%$ of the peak value. We normalize the input data to unit intervals in flux and time for consistent treatment of numerical precision. For a system modeled with $N_{\text {image }}$ images, then the $N_{\text {image }}-1$ relative time delays and $N_{\text {image }}-1$ relative magnifications are derived from $N_{\text {image }}$ absolute time positions and $N_{\text {image }}$ magnifications.

We infer the time delays and relative magnifications using the NoU-Turn Hamiltonian Monte Carlo (HMC) sampler within CMDSTAN, the command-line interface to the Stan statistical modeling language (Stan Development Team 2021). Our typical HMC run includes 8 chains, each with 1400 iterations and 250 warm up steps, with a system taking $\sim 0.4-4$ hours per chain, depending on the number of parameters (images) fit.

\subsection{Comparison with Crossing Method}

Advantages exist for each one of the fitting methods we apply to lensed lightcurves. The method of Paper 1, which we call the crossing method, allows the amplitude of deviation from the base form to vary widely, but places some constraints on the shape deviations. The freeform method here allows the shape of the lightcurve to vary widely but bounds the amplitudes of deviation. Allowing both shape and amplitude to vary without constraint is untenable as strong degeneracies are introduced such that, for example, an unlensed SN could be made to look like a blend of two images. We believe both the freeform and crossing method are useful, and provide valuable crosschecks.

We begin the comparison between the two by considering the twoimage systems used in Paper 1 for blind testing. By fitting them with the freeform technique we can directly compare the results.

Figure 1 shows the results for this paper's freeform technique (left) and Paper 1's crossing technique (right) for those 100 systems. The top panels show $\Delta t_{\text {fit }}$ vs $\Delta t_{\text {true }}$, the middle panels show the cumulative distribution function plots, and the lower panels the $\Delta t_{\text {fit }}-\Delta t_{\text {true }}$ histograms.

For $\Delta t_{\text {fit }}$ vs $\Delta t_{\text {true }}$, both techniques do quite well for $\Delta t_{\text {fit }} \geq 10$ days, tightly following the diagonal, i.e. truth. The freeform method continues to follow the diagonal below 10 days, down to about 6 days (this was the limit of the Paper 1 testing; in Appendix B here we see it actually works well to 2 days), while the crossing technique had leveled off with $\Delta t_{\text {fit }}=10$ (all $\Delta t$ are given in units of days). Also, for the unlensed systems $\left(\Delta t_{\text {true }}=0\right)$, the freeform method seems to control the fit somewhat better, keeping false positives well below $\Delta t_{\text {fit }}=10$, unlike the crossing technique. This makes the freeform method appear promising for application to the four image fits of the next section.

For the cumulative distribution functions (CDF), the key characteristic is whether the distribution has slope one, i.e. the fit distribution follows the true distribution, in the central part of the diagram, indicating reliable fit uncertainties. We indicate the central $68 \%$ probability distribution, between the 0.16 and 0.84 quantiles, by the vertical 

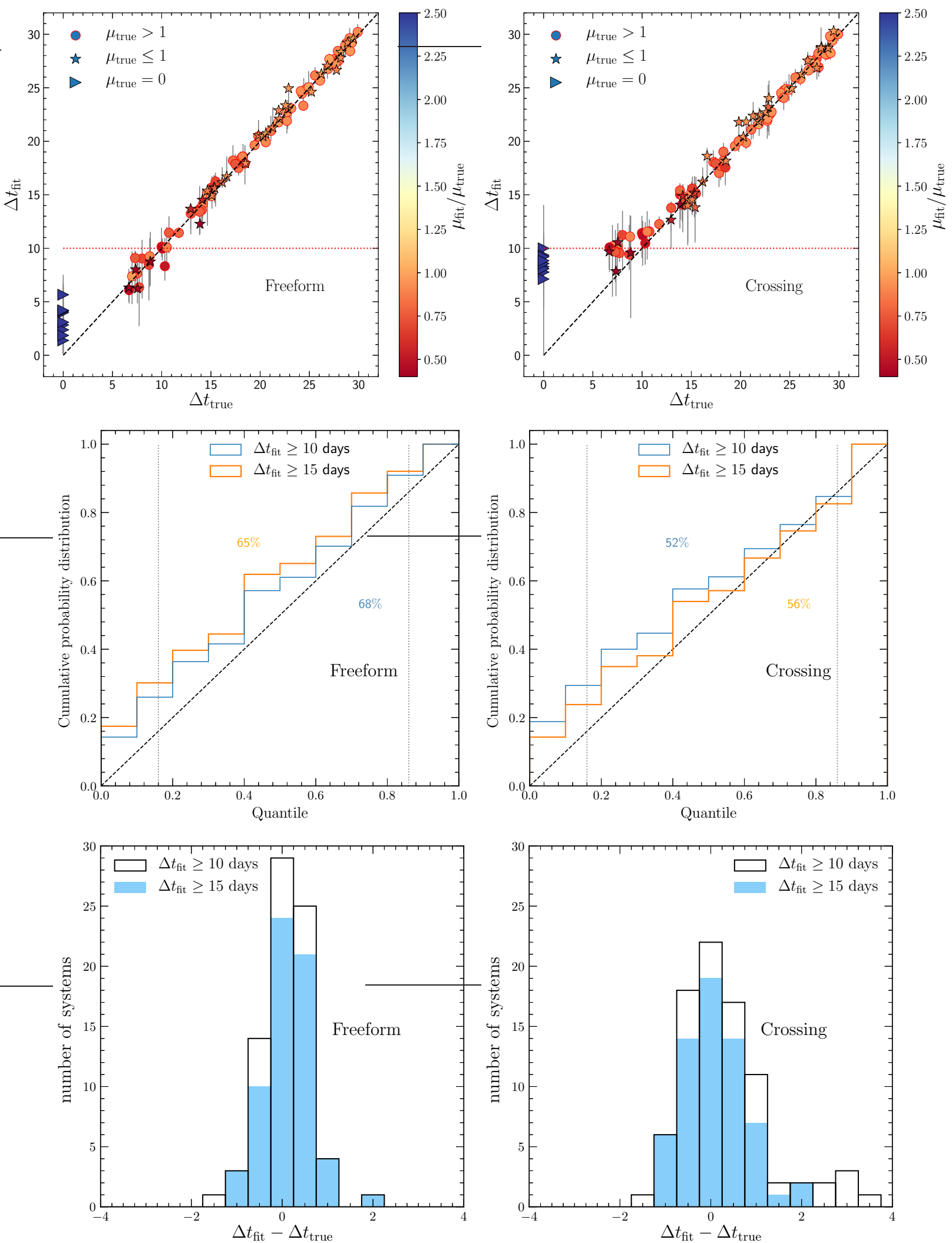

Figure 1. Blind tests using the freeform method (left panels) and crossing technique (right panels) on a set of 100 systems, with 9 unlensed and 91 two image systems with time delays are distributed over 6-30 days. [Top panels] Time delay fits lie closely along the diagonal $\Delta t_{\text {fit }}=\Delta t_{\text {true }}$. For $\Delta t_{\text {fit }}<10$ days, the crossing technique of Paper 1 tends to level out, while the freeform method continues to follow the diagonal. Unlensed systems $\left(\Delta t_{\text {true }}=0\right)$ are also clearly distinct from $\Delta t_{\text {fit }} \geq 10$ fits with the freeform method. Error bars show the $95 \%$ confidence intervals. [Middle panels] The cumulative distribution functions are shown for the systems with $\Delta t_{\text {fit }} \geq 10$ (blue) and $\Delta t_{\text {fit }} \geq 15$ (orange) days. The central $68 \%$ of fit probability encompasses $65-68 \%$ probability in the freeform case, showing excellent accuracy, an improvement over the 52-56\% of the crossing technique. [Bottom panels] Histograms of the time delay fits vs truth. Both show good peakiness around the truth. 
dotted lines. In that central $68 \%$ probability, the freeform method fits occur $65-68 \%$ of the time (depending on the cutoff in $\Delta t_{\text {fit }}$ ), demonstrating its accuracy, while the crossing technique had $52-56 \%$. Note that the vertical offset from the diagonal is due to a sample selection bias due to the imposed cutoff in $\Delta t_{\text {fit }}$, so that quantile zero is shifted to $\mathrm{CDF}>0$; but again, the central part of the diagram is the most important.

The histograms of $\Delta t_{\text {fit }}-\Delta t_{\text {true }}$ in Fig. 1 for $\Delta t_{\text {fit }} \geq 10$ days show that the freeform approach accurately recovers the true time delays, with better than 1.25 day precision in $\sim 97 \%$ of the cases, with accuracy $\lesssim 0.1$ day. The freeform nature of the approach allows greater flexibility in the lightcurve shape for robustness and eliminates the bias seen for the crossing method when $10 \leq \Delta t_{\text {fit }}<15$.

Thus the freeform technique has shown good accuracy and precision on two image systems, motivating its use for four image systems as well. We note that both approaches have advantages in particular areas. The crossing technique from Paper 1 starts from a form that is merely a rise and fall of flux, i.e. a fairly generic transient, without assuming it comes from a SN Ia, while the freeform technique does use deviations around a Hsiao SN Ia lightcurve. We might expect the crossing technique to be useful for unclassified transients, and then the freeform method to be more accurate for those suspected of being SN Ia. In the remainder of the paper we assume we are dealing with potentially lensed (one, two, or four images) SN Ia and employ the freeform method.

\section{TIME DELAYS OF FOUR IMAGES}

Having established that the freeform method works well on two image systems, and distinguishes them from unlensed $\mathrm{SN}$, we now proceed further and consider four image systems (recall that strong lensing generally produces two or four visible images, not an odd number).

For four image systems there are four (unobservable) times $t_{1}, t_{2}$, $t_{3}, t_{4}$ associated with the four images, and three observable, independent relative time delays, $\Delta t_{A B}, \Delta t_{B C}, \Delta t_{C D}$. We use the numerical 1-4 and alphabetical $A-D$ notation to emphasize the difference between unobservables and observables. Furthermore, there are four magnifications relative to the unlensed source flux, so there are a large number of permutations for the test system parameters. We therefore carry out two types of studies, one with systematic time delays and one with random time delays within a range.

First we systematically study the accuracy of four image fits by considering equal true delays: $\Delta t_{A B}=\Delta t_{B C}=\Delta t_{C D} \equiv \Delta T$ for $\Delta T=$ $4,6,8,10,12$ days. We still fit for each time delay as independent free parameters. These systems are simulated with a flux noise level of $5 \%$ of peak flux and a variety of image magnifications.

Figure 2 shows the fits, relative to truth, for the three relative time delays $\Delta t_{A B}, \Delta t_{B C}, \Delta t_{C D}$ for each of ten four-image systems (we choose ten systems randomly, enough to be statistically informative while not swamping the figure with $100+$ data bars). We exhibit the cases for $\Delta T=6$ (left panel) and $\Delta T=10$ (middle panel). While the fits have more variation than those for two images, nearly all contain the true value within the $95 \%$ uncertainties. Even for $\Delta T=6$ (well below what we will use), there is clear recognition that it is lensed at some nonzero time delay.

While we can fit four images well, plus distinguish them from the unlensed (zero time delay) case, we also want to see if we can distinguish them from the two image, lensed case. We therefore take the same systems and fit them as two image systems as well, i.e. with a single relative time delay and a relative magnification.

Figure 3 evaluates the relative $\chi^{2}$ of the fit with four images rela- tive to the fit with two images, as a function of true (four images) time delay spacing $\Delta T$. If $\Delta \chi^{2} \equiv \chi_{\min , 4 \mathrm{im}}^{2}-\chi_{\min , 2 \mathrm{im}}^{2}<0$, this means the four image fit is preferred. However, as the four image fit has four more parameters (two more time delays and two more relative magnifications), one might also look at the Akaike Information Criterion AIC $=\Delta \chi^{2}-2 \Delta N_{\text {dof }}$. Then four images is robustly preferred when $\Delta \chi^{2}<-8$. These two criteria are shown as the dotted red and dashed magenta horizontal lines, respectively. We see that for $\Delta T>8$ days we can have significant confidence that we can distinguish four image from two image lensed systems.

For the second study, we focus on time delays randomly distributed in the range $\Delta t_{i}=[10,14]$, where the subscript runs over $A B, B C$, $C D$. While we have seen that we can fit well smaller time delays for four image systems, and for two image systems, we will not have as robust confidence that we can tell the two cases apart. Therefore we concentrate on this range, which should be the most challenging of the fits for which we have confidence not only in the fits themselves but the number of images as well (see Section 4 for further investigation). We also randomly select the magnifications $\mu_{i}$.

We set flat priors on $t_{1}$ and $t_{4}$. The lower bound on $t_{1}$ is given by when the flux in $g$ band reaches $15 \%$ of flux peak value, and the upper bound is determined from the flux peak position. Similarly, the upper bound on $t_{4}$ is when the flux reaches the $15 \%$ of the peak value on the tail of the observation interval. The bounds on $t_{2}$ and $t_{3}$, and the lower bound on $t_{4}$, are determined dynamically in the fit, subject to the inequalities

$t_{2}>\tau t_{1}, \quad t_{3}>\tau t_{2}, \quad t_{4}>\tau t_{3}$,

where the factor $\tau>1$ is used to preserve some separation between the images. That is, we don't want images lying right on top of each other and hence being degenerate with a lesser number of images. We find that $\tau=1.1$ works well.

For the magnification we impose $\mu_{i}>0.25$, since recall that $\mu_{i}=0$ means there is no image (so a four image system with two $\mu_{i}=0$ is really a two image system) and there is a perfect degeneracy for all values of that $\Delta t_{i}$, while for $\mu_{i}$ near zero such a low amplitude bump is degenerate with a local shape perturbation $h(t)$. Thus the condition $\mu_{i}>0.25$ is employed to avoid these issues and enable robust fit convergence. The upper bound is $\mu_{i}<4$.

The right panel of Fig. 2 illustrates some time delay fits relative to the truth for these varying delay systems. The best fits are scattered about the truth, with no strong correlation between different time delays in a system, i.e. if one is on the positive side, the others may be on the positive or negative side. (More quantitatively, the correlation coefficients are $\lesssim 0.5$.) As for the previous study of equal time delays, the fits are well consistent with the truth. Figure 4 shows two examples of the four image fit to the observed lightcurve data, one with equal $\Delta T=12$ and one with time delays in the range $\Delta t_{i}=[10,14]$. The points with error bars are the observed data, with the thick solid line being the lightcurve constructed by the sum of the four image fits. The thin solid curves are the true image fluxes and the nonsolid curves the fit reconstructions - remember, the only observable is the data points of the unresolved, summed lightcurve. The fit looks quite good, with excellent $\chi^{2}$ and accurate time delay fits. The magnification fits are less good but not unreasonable. Our main focus however is identifying that an unresolved system is in fact a lensed system - out of one, many - and how many images, rather than optimizing per se the time delay and magnification estimation. 

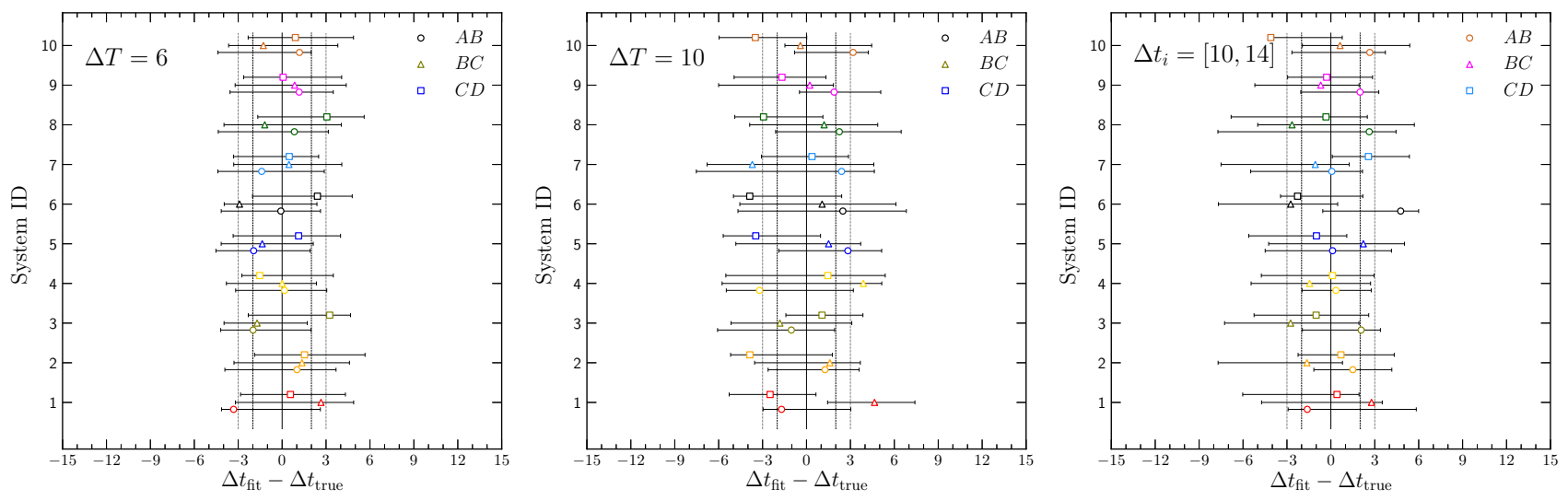

Figure 2. The time delay deviations from the truth in four-image systems for the equal time delay differences $\Delta T=6$ (left panel), $\Delta T=10$ (middle panel), and randomly chosen from the $\Delta t_{i}=[10,14]$ interval (right panel). The thin dashed and dotted vertical lines are used to indicate 2 and 3 day deviations respectively. Error bars show the $95 \%$ confidence intervals.

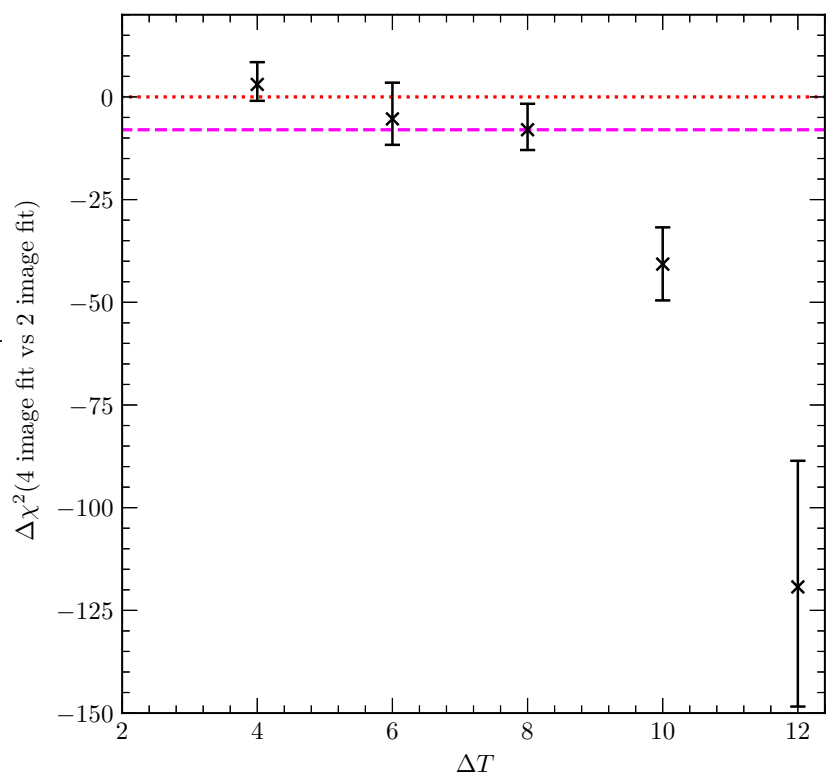

Figure 3. Distinction between systems with four images and with two images is statistically highly significant for time delay spacing of greater than eight days, generated for four image systems. Error bars give the mean $\Delta \chi^{2}$ difference and $68 \%$ confidence region at each $\Delta T$ spacing. Fits with $\Delta \chi^{2}<0$ (dotted red line) correctly prefer a four image fit, while those with $\Delta \chi^{2}<-8$ (dashed magenta line) have Akaike Information Criterion AIC $<0$, accounting for the four extra parameters of a four vs two image fit, and hence are fully robust.

\section{HOW MANY IMAGES?}

While obtaining four good image fits for data simulated with four images (or two for two) is important and valuable, we will not know the true number of images behind observed data (unlike for resolved lensed systems). Therefore it is essential that we be able to distinguish systems with one (unlensed) vs two vs four images. Fitting a system with the incorrect number of images will likely lead to either degeneracies or biases.

We have already explored this partially with Figure 3, and found robust distinction between four images and two images for $\Delta T \gtrsim 8$ days. Now we examine this more thoroughly, forming a statistical

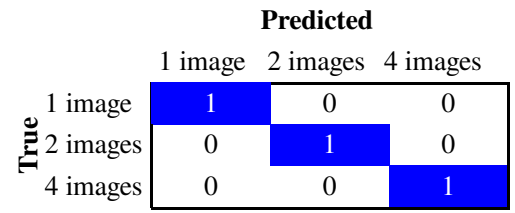

Table 1. The confusion matrix showing the performance of our one, two, four image models on a simulation set consisting of 100 unlensed systems, 100 2-image and 1004 -image lensed systems. The lensed systems have true adjacent time delays random in $[10,14]$ and magnification ratios in $[0.5,1.5]$. The threshold is $\Delta t_{\text {fit }} \geq 10$ days.

confusion matrix between one (unlensed), two, and four image systems, for realizations of random time delays $\Delta t_{i}=[10,14]$ and magnifications.

We simulate one, two, and four image systems, 100 of each, and fit every one with one, two, and four images (e.g. not just true two with fit two, but true two with fit one, two, and four). By examining the $\Delta \chi^{2}$ minima, and AIC, between the $N$ and $M$ image fits, we can decide whether we can robustly identify the number of images, and determine the time delays for the optimized case. The results of the fitting to simulations is summarized in the confusion matrix, with entries for each case where there are $N$ images simulated, i.e. truth, and $M$ images fit, for $N, M=1,2,4$. We assign the system to the highest number of images for which the AIC $<0$ and the fit time delays of the images exceed a given threshold, e.g. $\Delta t_{\text {fit }} \geq 10$ days. The fraction of true systems in each image category fit is given in the matrix entries.

Table 1 shows the results for our fiducial threshold, $\Delta t_{\text {fit }} \geq 10$ days. The confusion matrix is perfectly diagonal, showing purity of classification. As we have already seen in the previous sections, the time delay fit accuracy is excellent as well.

Studying the results as we reduce the threshold below our fiducial, we see in Table 2 that we still identify the number of images in the system perfectly for a threshold of $\Delta t_{\text {fit }} \geq 8$ days. Note we do have $10 \%$ of two image systems that are fit as four image systems with one time delay greater than 8 days, but $0 \%$ with two time delays greater than 8 days, and hence would not identify this as a four image system above threshold. It is not until the threshold is lowered to $\Delta t_{\text {fit }} \geq 6$ days that the confusion matrix becomes offdiagonal, as seen in Table 3 .

Thus, the freeform method can successfully identify both the num- 

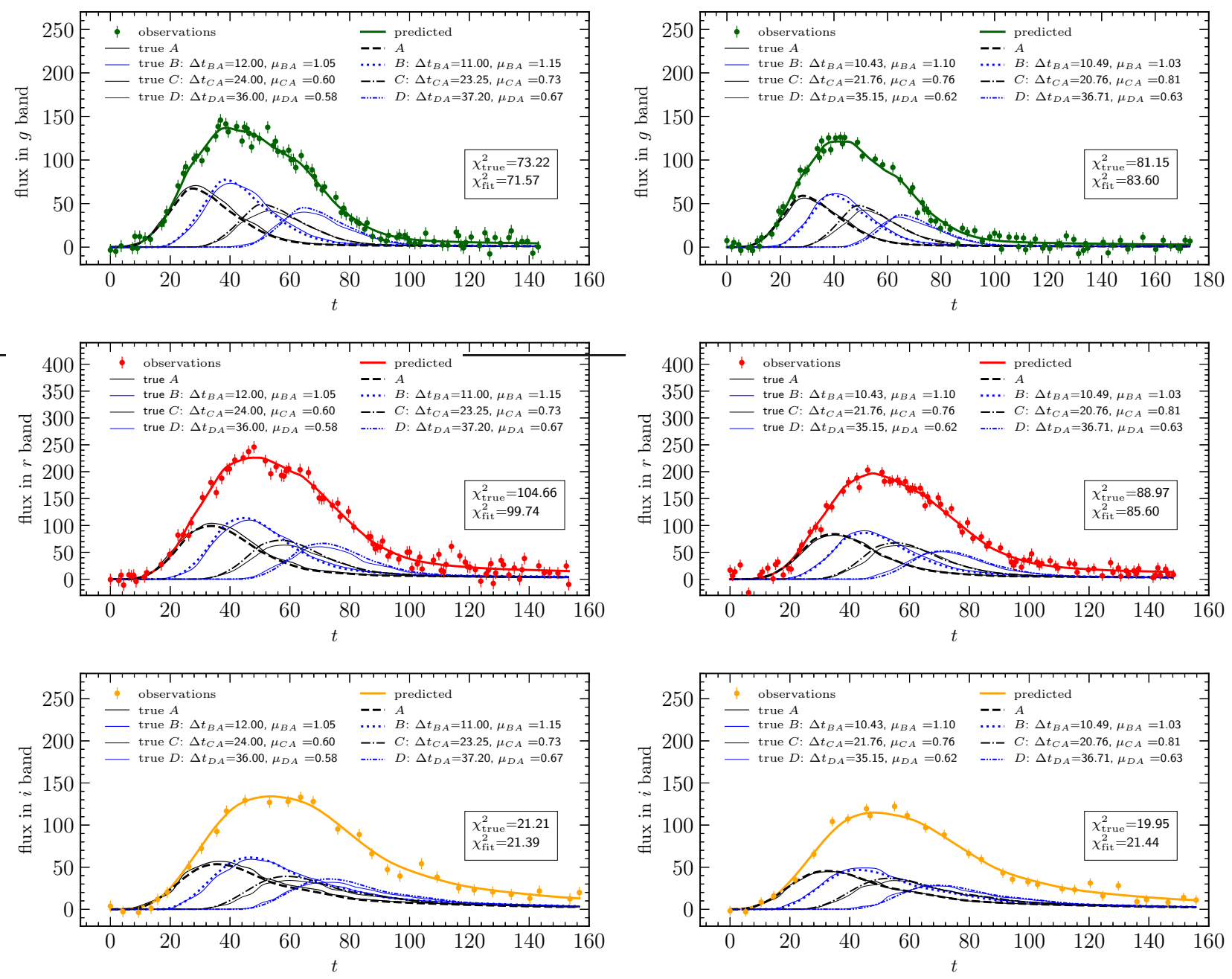

Figure 4. The simulated four-image data with $5 \%$ noise level are shown for two systems, with equidistant $\Delta T=12$ time delays (left column) and randomly distributed time delays in the range $\Delta t_{i}=[10,14]$ (right column). The upper, middle, and bottom rows show the lightcurves in $g, r, i$ bands respectively. The thin solid black and blue pairs of lines are the true light curves of individual images. The dashed, dotted, dash-dotted, and dash-double-dotted lines are the individual image flux reconstructions of our model using the best fit parameters. The extracted time delays and magnification ratios as well as their true counterparts are indicated in each panel. The $\chi^{2}$ values in the boxed texts indicate how well the reconstructed lightcurves fit the observations in comparison to the true ones.

Predicted

1 image 2 images 4 images

\begin{tabular}{l|ccc|}
1 image & 1 & 0 & 0 \\
2 images & 0 & 1 & 0 \\
4 images & 0 & 0 & 1 \\
\cline { 2 - 4 } & &
\end{tabular}

Table 2. As Table 1 but with a threshold of $\Delta t_{\text {fit }} \geq 8$ days.

\section{Predicted}

\begin{tabular}{c|ccc|} 
& 1 image & 2 images & 4 images \\
\hline 1 image & 1 & 0 & 0 \\
2 images & 0 & 0.97 & 0.03 \\
4 images & 0 & 0 & 1 \\
\cline { 2 - 4 } & & &
\end{tabular}

Table 3. As Table 1 but with a threshold of $\Delta t_{\text {fit }} \geq 6$ days.

ber of unresolved images, and the individual image time delays, for $\Delta t_{\text {fit }} \gtrsim 10$ days.

\section{CONCLUSIONS}

Lensed Type Ia supernovae have the potential to become a significant new cosmological probe with the new generation of cosmic surveys. The time delays between images carry information on the scale and expansion history of the universe. However, not all multiply imaged $\mathrm{SN}$ will have visibly and resolved multiple images from which to measure the time delays. Images may be unresolved or blended, with a single measured lightcurve summing over the images. We investigate the problem of how to get out of one, many - detecting the actual number of images and measuring their time delays.

In Paper 1 we demonstrated one successful approach on two image systems. While it allowed wide variations in amplitude, it placed constraints on the shape of the lightcurve, correlated over its rise and fall. Here we include four image systems, where the variations in shape can be significant, so we develop and validate a new fitting method, essentially freeform in shape but more limited in amplitude variations. Neither method assumes a fixed template for the lightcurve. 
The freeform method performs quite well for time delays $\Delta t \gtrsim 10$ days, and has reasonable behavior for $\Delta t \lesssim 10$ days as well. We then address the question of multiplicity, distinguishing not only whether a source has been lensed, but accurately discerning the number of images, while simultaneously fitting for their time delays (and to a lesser extent their magnifications). The confusion matrix - detailing whether a system is correctly identified or whether there are false negatives or positives - is nearly diagonal, i.e. pure, for the freeform method for $\Delta t \gtrsim 10$ days, and does well for shorter time delays as well.

The goal of this research is to establish the ability to achieve out of one, many, to go as far as possible with unresolved but potentially blended lightcurves, in order to productively select systems for followup. That followup, e.g. obtaining resolved images through higher resolution imaging (and further ingredients such as for lens modeling) can turn the time delay systems into incisive cosmological probes.

Our approach avoids fixed templates in the hope of eventually being applicable to a wide range of transients, beyond Type Ia supernovae. While the crossing technique in Paper 1 is particularly useful to deal with unclassified lensed transients, the freeform method used here is seen to be more precise and accurate for those suspected of being SN Ia that are of crucial importance in cosmology. A combination of these two approaches and crosschecks between their results offer the potential to deal with a broad range of transients. (While we focus on transients, combined lightcurves from quasars have been studied by, e.g., (Shu et al. 2021; Springer \& Ofek 2021b,a; Pindor 2005; Geiger \& Schneider 1996).)

Future research could include recognition of lensing for diverse transients, fitting of partial lightcurves (e.g. early times) or different cadence (we studied the effect of S/N in Paper 1, and for this article chose a conservative noise level), etc. We will seek to improve further our approaches to classify various lensed transients and estimate their time delays. An overall objective for the community is to determine accurate time delay distances for a large fraction of real systems (not just Type Ia supernovae). While a large sample is desired, our focus here emphasized an unbiased pure sample over completeness. We present a model that is designed to be accurate for transients with intrinsic lightcurves within $\sim 0.1$ mag of a base shape at independent phases, and can characterize lensed systems when the unresolved observed summed lightcurves deviate more than 0.1 mag from that single unlensed source lightcurve. From simulations, we find that accepting $\mathrm{SN}$ with $\Delta t_{\text {fit }} \gtrsim 10$ effectively removes lensing false positives. We leave to further work the development of methods that identify more general systems with significant $\Delta t$, but a very different shape, i.e. deviating by more than 0.1 mag from the base shape. Comparison of fits of multiple models, such as the crossing method and the freeform method using base shapes from different source classes, may be used to identify systems needing further examination.

In addition, Paper 3 (in draft) deals with the impact of microlensing on supernova time delay estimation; preliminary results show it appears tractable within the approaches of Paper 1 and this paper, while having two methods acts as a useful crosscheck.

\section{ACKNOWLEDGEMENTS}

We are grateful to Nazarbayev University Research Computing for providing computational resources for this work. This work was supported in part by the Energetic Cosmos Laboratory. AK and EL are supported in part by the U.S. Department of Energy, Office of
Science, Office of High Energy Physics, under contract no. DEAC02-05CH11231. AS would like to acknowledge the support by National Research Foundation of Korea NRF-2021M3F7A1082053, and the support of the Korea Institute for Advanced Study (KIAS) grant funded by the government of Korea.

\section{DATA AVAILABILITY}

The data for the 300 simulated lightcurves of 1, 2, and 4 image systems, as used in the confusion matrix results, are available on the GitHub repository https://github.com/mdeatecl/LensedSN124imagesLCs. We include the LCsimulator code used to generate the lightcurves for this article in the same repository.

\section{REFERENCES}

Bag S., Kim A. G., Linder E. V., Shafieloo A., 2021, Astrophys. J., 910, 65 Barbary K., et al., 2020, SNCosmo: Python library for supernova cosmology. Package version 2.1, https://github.com/sncosmo/sncosmo

Bellm E. C., et al., 2018, Pub. Ast. Soc. Pac., 131, 018002

Geiger B., Schneider P., 1996, Mon. Not. Roy. Astron. Soc., 282, 530

Goldstein D. A., Nugent P. E., Goobar A., 2019, Astrophys. J. Suppl., 243, 6 Guy J., et al., 2007, Astron. Astrophys., 466, 11

Hazra D. K., Shafieloo A., 2014, JCAP, 01, 043

Hsiao E. Y., Conley A. J., Howell D. A., Sullivan M., Pritchet C. J., Carlberg R. G., Nugent P. E., Phillips M. M., 2007, Astrophys. J., 663, 1187

Huber S., et al., 2022, A\&A, 658, A157

Liao K., Shafieloo A., Keeley R. E., Linder E. V., 2020, Astrophys. J. Lett., 895, L29

Linder E. V., 2004, Phys. Rev. D, 70, 043534

Linder E. V., 2011, Phys. Rev. D, 84, 123529

Millon M., et al., 2020, Astron. Astrophys., 639, A101

Pierel J. D. R., Rodney S., Vernardos G., Oguri M., Kessler R., Anguita T., 2021, Astrophys. J., 908, 190

Pindor B., 2005, Astrophys. J., 626, 649

Refsdal S., 1964, Mon. Not. Roy. Astron. Soc., 128, 295

Rodney S. A., Brammer G. B., Pierel J. D. R., Richard J., Toft S., O’Connor K. F., Akhshik M., Whitaker K., 2021, Nature Astronomy, 5, 1118

Shafieloo A., 2012a, JCAP, 05, 024

Shafieloo A., 2012b, JCAP, 08, 002

Shafieloo A., Clifton T., Ferreira P. G., 2011, JCAP, 08, 017

Shajib A. J., et al., 2020, Mon. Not. Roy. Astron. Soc., 494, 6072

Shu Y., Belokurov V., Evans N. W., 2021, Mon. Not. Roy. Astron. Soc., 502, 2912

Springer O. M., Ofek E. O., 2021a, Mon. Not. Roy. Astron. Soc., 506, 864

Springer O. M., Ofek E. O., 2021b, Mon. Not. Roy. Astron. Soc., 508, 3166

Stan Development Team 2021, CMDStan: the shell interface to Stan. Package version 2.27, http://mc-stan.org/

Treu T., Marshall P. J., 2016, Astron. Astrophys. Rev., 24, 11

Wong K. C., et al., 2020, Mon. Not. Roy. Astron. Soc., 498, 1420

\section{APPENDIX A: TESTING FREEFORM WITH SALT2}

The Hsiao template is used in two ways: to generate the simulated systems, and also as the base from which the freeform method starts in allowing shape deviations. We want to assess whether this affects the fits, despite the addition of 50-70 free parameters allowing a free form. To do so, we generate systems using the SALT2 lightcurve template Guy et al. (2007) instead, and fit them with the freeform method acting on the Hsiao base, i.e. Eq. (2).

Figure A1 shows that the freeform fit works comparably well 

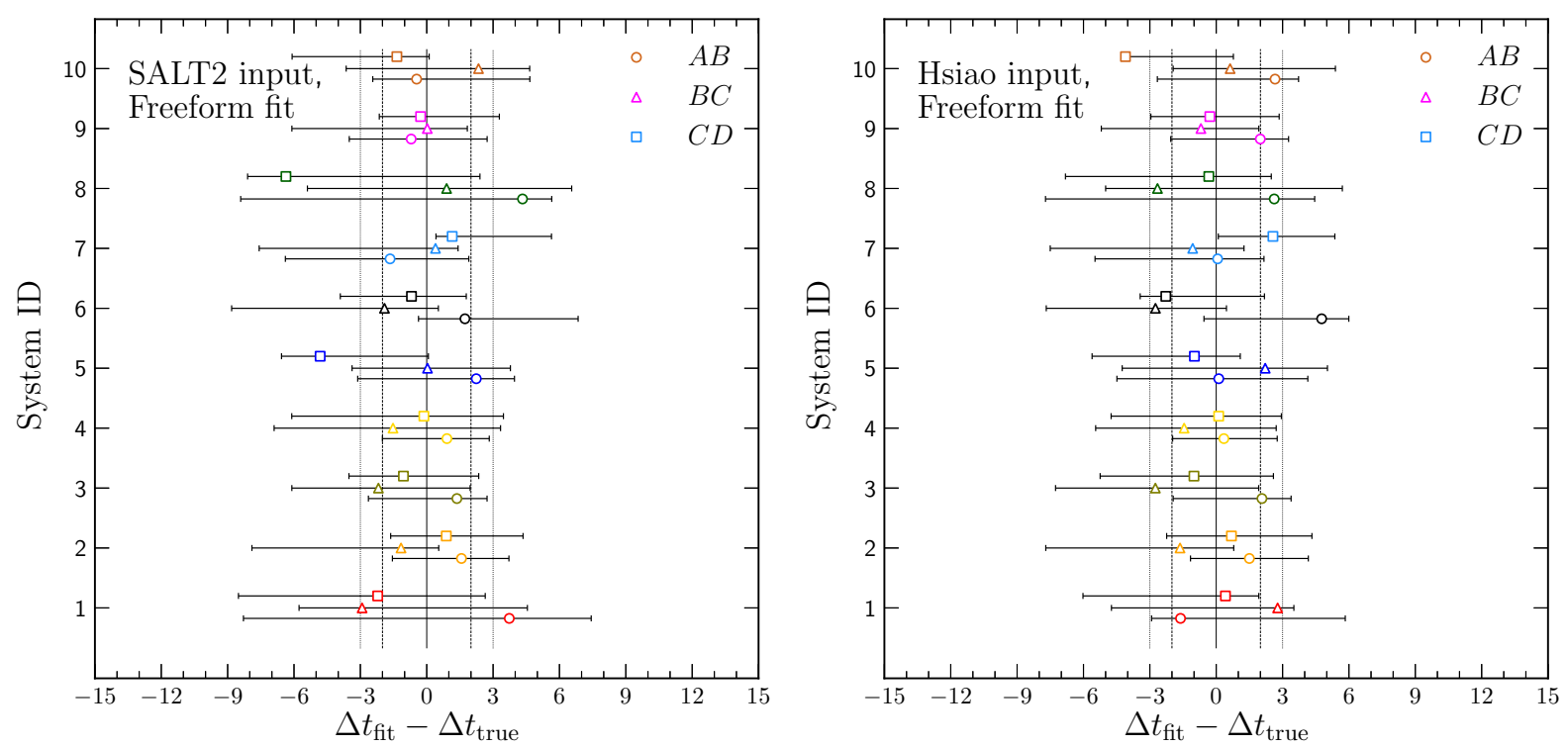

Figure A1. The time delay deviations from the truth in four-image systems generated using the SALT2 (left panel) or Hsiao (right panel; as the right panel in Fig. 2) templates and fitted using the freeform method with the Hsiao base. The time delays are randomly chosen from the $\Delta t_{i}=[10,14]$ interval. The freeform fit works even with a different supernova input form. Error bars show the $95 \%$ confidence intervals.

whether the data is generated based on SALT2 or Hsiao. As a further check we have verified that the clear distinction between four image and two image systems continues to hold with the freeform method even when generated with SALT2.

\section{APPENDIX B: ESTIMATING 2-IMAGE TIME DELAYS BELOW 8 DAYS}

As seen in Fig. 1 for two image systems, the freeform method continues to yield $\Delta t_{\text {fit }} \approx \Delta t_{\text {true }}$ below the fiducial threshold of $\Delta t_{\text {fit }}=10$ days. Although we do not use systems with shorter time delays in the main text, here we explore two image time delays with $2 \leq \Delta t_{\text {true }}<8$ days. Figure B1 shows that the fits continue to follow $\Delta t_{\text {fit }} \approx \Delta t_{\text {true }}$, with scatter of approximately two days (comparable to the observation cadence). However, also as seen in Fig. 1, we expect unlensed systems to "upscatter" into this region of $\Delta t_{\text {fit }}$ and so to avoid false positives we conservatively do not use a lower threshold $\Delta t_{\text {fit }}<10$.

This paper has been typeset from a $\mathrm{T}_{\mathrm{E}} \mathrm{X} / \mathrm{L} \mathrm{T}_{\mathrm{E}} \mathrm{X}$ file prepared by the author.

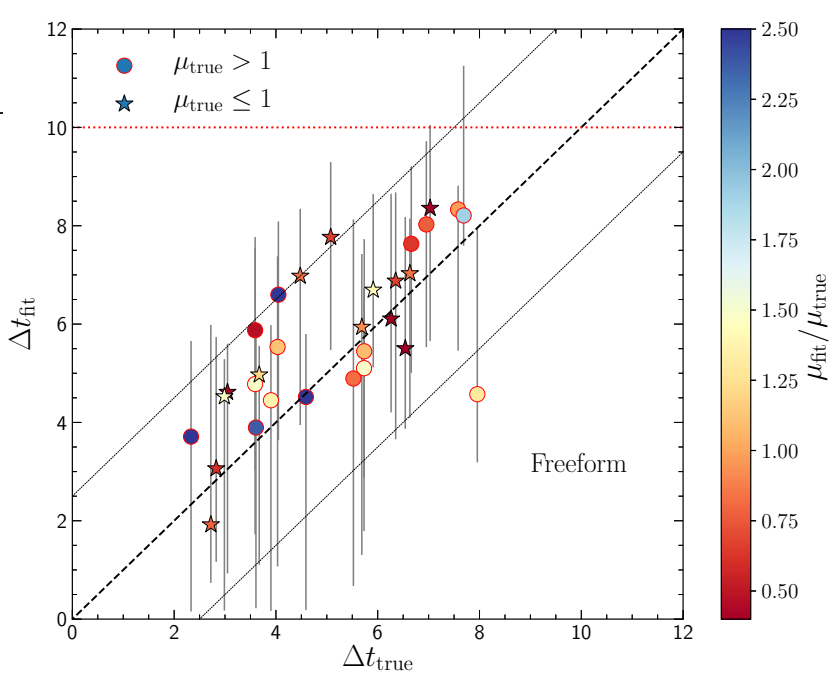

Figure B1. Time delay fits are given for 30 two image systems, showing that $\Delta t_{\text {fit }} \approx \Delta t_{\text {true }}$ even in the regime $2 \leq \Delta t_{\text {true }}<8$ days, below the threshold used in the main text. The dashed diagonal line gives $\Delta t_{\text {fit }}=\Delta t_{\text {true }}$, while the dotted diagonals give \pm 2.5 days around this. Error bars show the $95 \%$ confidence intervals. 